\title{
Novel linkage disequilibrium clustering algorithm identifies new lupus genes on meta-analysis of GWAS datasets
}

\author{
Mohammad Saeed $^{1}$ (D) \\ Received: 15 December 2016 / Accepted: 13 February 2017 / Published online: 28 February 2017 \\ (C) The Author(s) 2017. This article is published with open access at Springerlink.com
}

\begin{abstract}
Systemic lupus erythematosus (SLE) is a complex disorder. Genetic association studies of complex disorders suffer from the following three major issues: phenotypic heterogeneity, false positive (type I error), and false negative (type II error) results. Hence, genes with low to moderate effects are missed in standard analyses, especially after statistical corrections. OASIS is a novel linkage disequilibrium clustering algorithm that can potentially address false positives and negatives in genome-wide association studies (GWAS) of complex disorders such as SLE. OASIS was applied to two SLE dbGAP GWAS datasets (6077 subjects; $~ 0.75$ million single-nucleotide polymorphisms). OASIS identified three known SLE genes viz. IFIH1, TNIP1, and CD44, not previously reported using these GWAS datasets. In addition, 22 novel loci for SLE were identified and the 5 SLE genes previously reported using these datasets were verified. OASIS methodology was validated using single-variant replication and gene-based analysis with GATES. This led to the verification of $60 \%$ of OASIS loci. New SLE genes that OASIS identified and were further verified include TNFAIP6, DNAJB3, TTF1, GRIN2B, MON2, LATS2, SNX6, RBFOX1, $N C O A 3$, and $C H A F 1 B$. This study presents the OASIS algorithm, software, and the meta-analyses of two publicly available SLE GWAS datasets along with the novel SLE genes. Hence, OASIS is a novel linkage disequilibrium clustering
\end{abstract}

Electronic supplementary material The online version of this article (doi:10.1007/s00251-017-0976-8) contains supplementary material, which is available to authorized users.

Mohammad Saeed

saeed.khan@arkanalabs.com

1 Department of Genomics, Arkana Laboratories, 10810 Executive Center Drive, Suite 100, Little Rock, AR 72211, USA method that can be universally applied to existing GWAS datasets for the identification of new genes.

Keywords Lupus · Linkage disequilibrium · Genome-wide association study $\cdot$ Gene-based tests $\cdot$ Meta-analysis

\section{Introduction}

Complex disorders such as systemic lupus erythematosus (SLE) could be thought of as a mixture of multiple resembling phenotypes, each a result of a separate mutation in genes of a causal pathway (Saeed 2017). Finding a particular gene then depends on the enrichment of a causal mutation carrying haplotype in the study sample. In a genome-wide association study (GWAS), hundreds of thousands of genetic markers are typed creating a multiple testing problem. As a result of random noise, true association signal is hard to decipher. To reduce this error (type I), corrective measures such as the Bonferroni are applied. These may be overly corrective and prevent identification of true associations (type II error), leading to increase in study sample sizes and consequent expense.

GWAS is based on the principle that complex disorders are caused by common variants (frequency $>1 \%$ ) and should therefore be detectable by linkage disequilibrium (LD) mapping using a large number of common variants. Here, this principle is expanded upon by the development of a novel clustering algorithm to identify genes and loci of interest in SLE. As previously shown, gene- or region-based association analysis is an approach that may improve the power of GWAS and allow detection of genes of modest influence (Zhang et al. 2015). It is known that true genetic associations are accompanied by signals from surrounding markers; i.e., singlenucleotide polymorphisms (SNPs) in LD with the susceptibility mutation also have statistically significant $P$ values (Martin 
et al. 2000). Diagrammatically, these surrounding SNPs form a cluster around the causal variant, an "OASIS," observable in numerous GWAS (Duerr et al. 2006; Edwards et al. 2005; Rioux et al. 2007). Metaphorically, these clusters represent oasis in "gene deserts," a term usually used to describe absence of coding sequences in DNA; however, in the context of gene, hunting may represent absence of disease susceptibility genes. Hence, this algorithm is termed OASIS.

In this study, OASIS meta-analysis of two SLE GWAS datasets (Harley et al. 2008; Hom et al. 2008) identified three known SLE genes viz. IFIH1, TNIP1, and CD44 that were not identified in the original studies. OASIS verified the five genes either of the two studies identified, in both datasets viz. STAT1/STAT4, DNASE1L3/PXK, IRF5, BLK, and ITGAM/ITGAX. Furthermore, 22 new loci for SLE were identified. Of these, 10 genes were validated by standard singlevariant and/or gene-based replication. These new SLE genes include TNFAIP6, DNAJB3, TTF1, GRIN2B, MON2, LATS2, $S N X 6, R B F O X 1, N C O A 3$, and $C H A F 1 B$. Hence, OASIS is a novel LD clustering method that can be broadly used to mine existing GWAS datasets for new complex disease genes.

\section{Methods}

\section{Datasets}

GWAS datasets were obtained online from the publicly available dbGAP repository. Meta-analysis of two SLE datasets, phs000202 (Harley et al. 2008) and phs000122 (Hom et al. 2008), was conducted using OASIS. The dataset phs000202 consisted of 706 SLE females and 353 controls and was used for screening (Harley et al. 2008), while phs000122, comprising of 1435 SLE cases and 3583 controls genotyped for $500 \mathrm{~K}$ SNPs, was used as the replication dataset (Hom et al. 2008). The $P$ values reported in these datasets were based on standard association analysis results as described in the original studies (Harley et al. 2008; Hom et al. 2008).

\section{OASIS algorithm}

In the OASIS algorithm, LD clusters were defined by $200 \mathrm{~kb}$ regions. This cutoff has previously been used to define an LD cluster (Bentham et al. 2015). The GWAS file was ordered sequentially by chromosomes and position. The first variant that had a $P \leq 0.05$ was considered the start of a new OASIS block. SNPs with $P \leq 0.05$, located within $200 \mathrm{~kb}$ of this initial

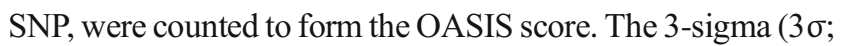
three standard deviations or a value $\geq 99.7 \%$ of the data) cutoffs were calculated for the $-\log [\mathrm{P}]$ values and the OASIS scores. This structured the data in two axes $(-\log [\mathrm{P}]$ and OASIS) and four groups viz. quadrants A-D (Fig. S1). Quadrant A loci were those that crossed the $3 \sigma$ cutoffs on both axes, quadrant
B loci were positive on $-\log [\mathrm{P}]$ but not on OASIS, quadrant $\mathrm{C}$ loci were positive on OASIS scores but not on $-\log [\mathrm{P}]$, whereas quadrant $D$ loci failed to meet the $3 \sigma$ cutoffs on either axis.

\section{OASIS software}

The code has been written in Python 2.7.9 (https://github.com/drsaeed/OASIS/blob/master/OASIS.py) and comprises of three modules. Module 1 reads the GWAS data file and calculates the OASIS scores which are processed by Module 2 to generate the $3 \sigma$ statistics and graphs (saved in PNG format), for $-\log [\mathrm{P}]$ values, OASIS scores, and quadrants $\mathrm{A}-\mathrm{D}$ for each chromosome (Fig. S1). The software can be used for analysis using varying OASIS block sizes, though $200 \mathrm{~kb}$ is set as default. GWAS data from dbGAP as well as PLINK output files can be analyzed using the OASIS software. Module 3 is for creating a composite of two GWAS datasets into a single html table with clickable links to NCBI Mapview for easy location of associated regions. Module 3 highlights the overlapping $3 \sigma$ significant regions in the two datasets with information on their respective quadrants, maximum $-\log [\mathrm{P}]$ values in those regions, and OASIS scores, besides other valuable data in a tabulated scrollable format. LD has been previously shown to maximally extend to about $2 \mathrm{Mb}$ (Saeed et al. 2009). Therefore, loci overlapping within $2 \mathrm{Mb}$ distance were considered replicated in Module 3 analyses. Moreover, this allowed a reasonable comparison between GWAS datasets, which often use different genotyping platforms.

\section{OASIS validation using standard analysis}

Single-variant replication was performed on SNPs with maximum $-\log [\mathrm{P}]$ values in loci identified by OASIS. These SNPs in the dataset phs000202 (Harley et al. 2008) were verified for association in the phs 000122 (Hom et al. 2008) dataset. Gene-based replication was performed using Genebased Association Test using Extended Simes procedure (GATES) (Li et al. 2011) as implemented in the KGG software (Li et al. 2010). SNPs were mapped onto genes according to positional information from the NCBI GRCh37 database, and SNPs within $10 \mathrm{~kb}$ upstream and $10 \mathrm{~kb}$ downstream of each gene were included as well (Zhang et al. 2015). OASIS candidate genes were used as the seed list for GATES verification.

\section{Results}

\section{Linkage disequilibrium clustering (OASIS)}

Data for 258,357 and 489,876 SNPs was available from dbGAP for the datasets phs000202 and phs000122, respectively (Harley et al. 2008; Hom et al. 2008). The input data included information on chromosome number, SNP, location, 
and $P$ value. On OASIS analysis, 5082 regions in the phs000202 dataset were identified, which had at least one variant with a $P \leq 0.05$. Similarly, 6342 such OASIS blocks were identified in the dataset phs000122. Of these, 292 loci crossed the $3 \sigma$ cutoffs and were classified in quadrants A, B, or C (159 from the dataset phs000202 and 133 from phs000122). OASIS Module 3 analyses showed that 34 blocks replicated in both datasets. A locus was considered replicated when at least two OASIS blocks from separate datasets were located less than $2 \mathrm{Mb}$ apart. Some of these blocks overlapped, resulting in the identification of a total of 30 SLE loci containing 80 candidate genes (Table S1).

The HLA locus on chromosome 6 showed the highest significance in both datasets on OASIS as well as $-\log [\mathrm{P}]$ analysis (Fig. 1a, b), dwarfing other signals. This affected the $3 \sigma$ cutoff values, and a second OASIS analysis for non-HLA loci was performed after removing the variants in the HLA locus $(25-34 \mathrm{Mb})$. It is this analysis that resulted in the identification of 292 loci mentioned above (Fig. 2a, b).

In the original studies, five genes/loci were identified using these datasets (Harley et al. 2008; Hom et al. 2008). STAT1/STAT4, ITGAM/ITGAX loci, and IRF5 were found to be associated with SLE in both studies (Harley et al. 2008; Hom et al. 2008). These were verified by OASIS as well. However, DNAse1L3/PXK locus was originally identified using the dataset phs000202 (Harley et al. 2008) and $B L K$ was identified only in the dataset phs000122 (Hom et al. 2008), while OASIS identified these loci in both datasets (Table S1). BLK was identified in quadrant $\mathrm{B}$ and replicated in quadrants $\mathrm{A}$ and $\mathrm{C}$.

a

Chromosome: 6
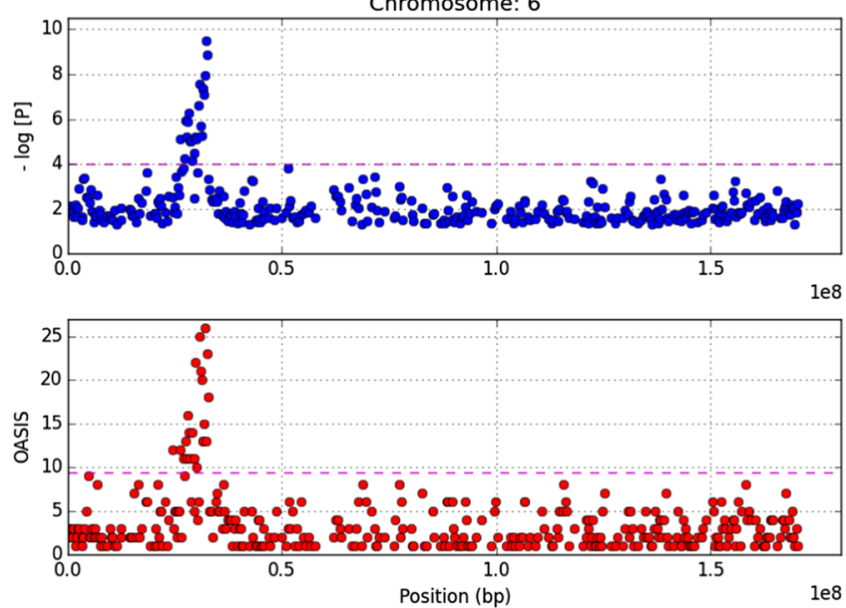

Note:

A - OASIS screening analysis with dataset phs000202 7

B - OASIS replication analysis with dataset phs $000122^{8}$

Fig. 1 a, b HLA association with SLE on chromosome 6 shows the high association signal $(-\log [\mathrm{P}]$ and OASIS score $-y$ axes $)$ on chromosome 6 (SNP position in base pairs $-x$ axis) for data from both SLE GWAS
DNAse1L3/PXK locus was found to be significant in quadrant $\mathrm{B}$ in both screening and replication datasets. This shows that the reason these two loci were missed in one of the studies was due to the use of the stringent Bonferroni correction. Moreover, this finding verifies the application of the $3 \sigma$ rule to GWAS data.

The success of the OASIS methodology is demonstrated by the identification of known SLE genes not identified using these datasets in the original studies. IFIHI screened positive in quadrant $\mathrm{C}$ and replicated in quadrant B (locus 2; Table S1). This shows that the OASIS algorithm based on LD clustering is valid, since IFIH1 shown to be associated using standard analysis in several separate later datasets could not be previously identified in the datasets used here (Gateva et al. 2009; Robinson et al. 2011; Wang et al. 2013). Verification in quadrant B again strengthens the concept of using the $3 \sigma$ rule. Of even greater significance was the identification, using these GWAS datasets, of the known SLE genes TNIP1 (locus 7) and CD44 (locus 15). Both these genes could not be identified in these datasets using standard association analysis, though they have been shown to be associated with SLE in later studies (Gateva et al. 2009; Lessard et al. 2011; Sheng et al. 2015; Yung and Chan 2012; Ramos et al. 2011). However, using the novel OASIS algorithm, these genes were identified and replicated in quadrant $\mathrm{C}$, indicating the sheer usefulness of this analytic technique.

The above findings lend support to the 22 novel SLE loci that were found using OASIS (Table S1). Interesting

b
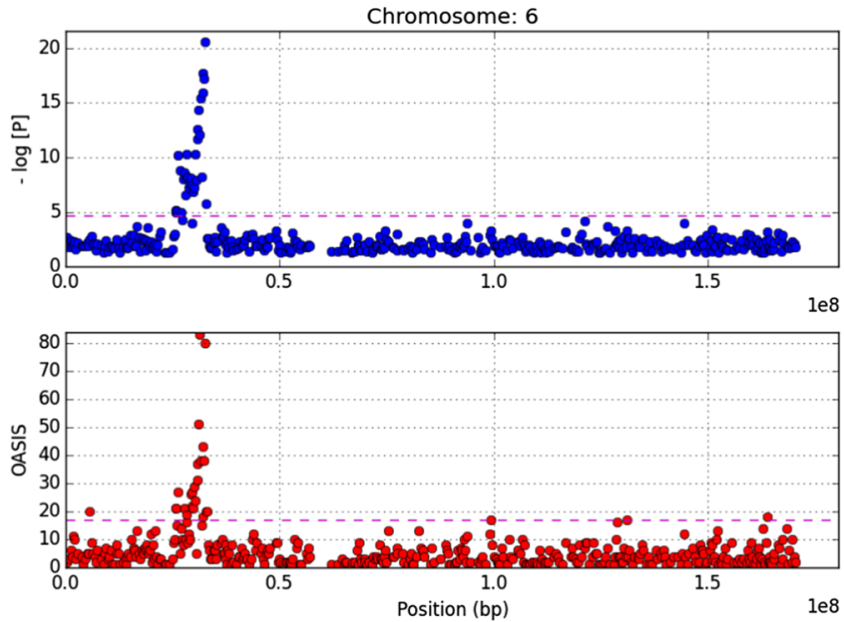

studies (Harley et al. 2008; Hom et al. 2008). OASIS software automatically generates these graphs as PNG and marks the $3 \sigma$ cutoff values with a mauve dashed line on the $y$ axes 
a

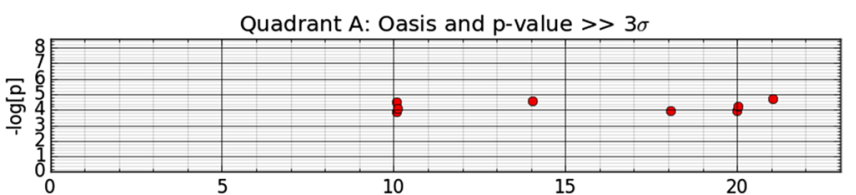

Quadrant B: p-value $>>3 \sigma$

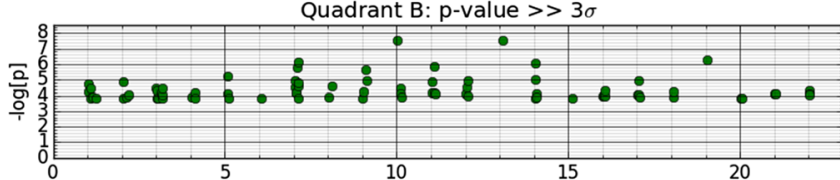

Quadrant C: Oasis $>3 \sigma$

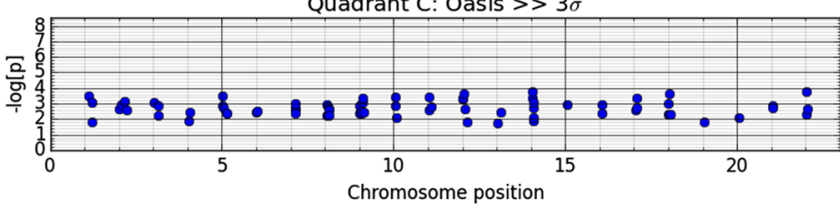

Note:

A - OASIS screening analysis with dataset phs000202 ${ }^{7}$

B - OASIS replication analysis with dataset phs $000122^{8}$

Fig. 2 a, b OASIS genome-wide association in the two SLE datasets shows the $-\log [\mathrm{P}]$ values for variants across the genome according to their categorization in quadrants $(\mathrm{A}, \mathrm{B}$, or $\mathrm{C})$ in the two SLE datasets

candidate genes included TNFAIP6 (locus 1), DNAJB3 (locus 4), TTF1 (locus 13), MON2 (locus 20), LATS2 (locus 21), RBFOX1 (locus 26), NCOA3 (locus 29), and CHAF1B (locus 30). Besides their potential pathogenic significance to SLE, the association signals at these loci were mostly concentrated in a narrow region around these genes signifying strong focal LD. The LATS2 and $C H A F 1 B$ loci are of particular interest because they were either identified or replicated in quadrant A. Most other loci were found in quadrant B or C. Interestingly, LATS2 was identified in quadrant $C$ and replicated in quadrant $A$ and vice versa for $C H A F 1 B$. These findings further strengthen the LD-based clustering approach of OASIS. b
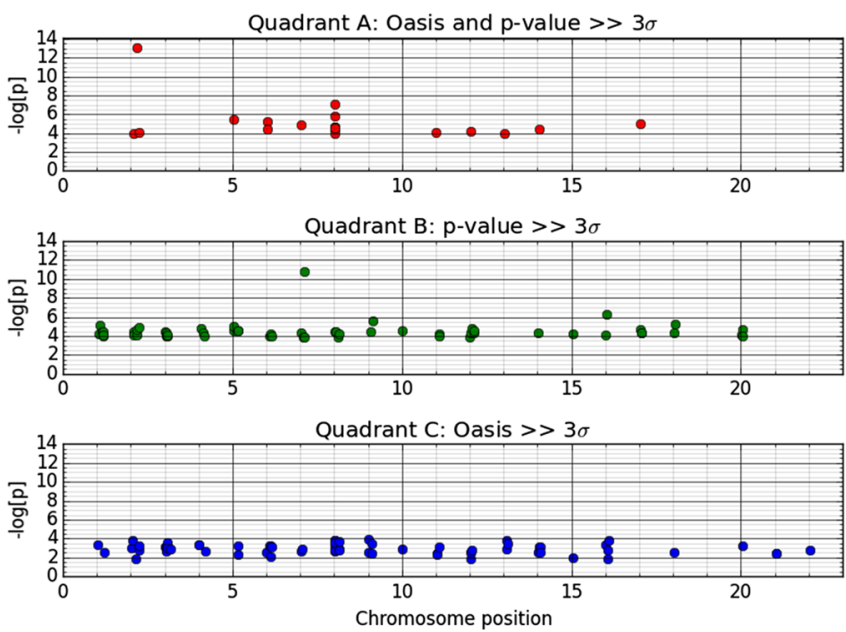

(Harley et al. 2008; Hom et al. 2008). This is based on the intersection of $-\log [\mathrm{P}]$ and OASIS score for each variant

\section{Single-variant replication}

To validate the loci identified by OASIS in a standard association study model, SNPs that showed the maximum $-\log [\mathrm{P}]$ values in the OASIS analysis of dataset phs000202 (Harley et al. 2008) were subjected to replication in the phs000122 (Hom et al. 2008) dataset. As shown in Table 1, 7 of 34 SNPs replicated. Of the genes identified in the original studies (Harley et al. 2008; Hom et al. 2008), SNPs in IRF5, ITGAM/ITGAX, and STAT1/ STAT4 replicated, while those in the BLK and DNASe1L3/ $P X K$ did not. The SNP, rs 2785197 , with the highest $-\log$ [P] value in the OASIS locus for CD44, a known SLE
Table 1 Single-variant replication of OASIS identified loci

\begin{tabular}{llllll}
\hline SLE gene (OASIS) & SNP ID & $P$ value & Rank & FDR & P-FDR \\
\hline IRF5 & rs12537284 & $5.50 \mathrm{E}-07$ & 1 & $1.47 \mathrm{E}-03$ & $1.47 \mathrm{E}-03$ \\
ITGAM, ITGAX & rs9888739 & $6.65 \mathrm{E}-07$ & 2 & $2.94 \mathrm{E}-03$ & $2.94 \mathrm{E}-03$ \\
IRF5 & rs4728142 & $1.46 \mathrm{E}-06$ & 3 & $4.41 \mathrm{E}-03$ & $4.41 \mathrm{E}-03$ \\
STATI, STAT4 & rs3771327 & $1.25 \mathrm{E}-03$ & 4 & $5.88 \mathrm{E}-03$ & $4.63 \mathrm{E}-03$ \\
CD44 & rs2785197 & $7.13 \mathrm{E}-03$ & 5 & $7.35 \mathrm{E}-03$ & $2.18 \mathrm{E}-04$ \\
RBFOX1 & rs1881335 & $3.34 \mathrm{E}-02$ & 6 & $8.82 \mathrm{E}-03$ & NS \\
MIRLET7I, PPM1H, MON2 & rs2704757 & $4.38 \mathrm{E}-02$ & 7 & $1.03 \mathrm{E}-02$ & NS \\
\hline
\end{tabular}

SNPs with the highest $-\log [p]$ values in the screening dataset (Harley et al. 2008) were verified in the replication dataset (Hom et al. 2008). Seven SNPs replicated at $p<0.05$ and five SNPs crossed the false discovery rate (FDR). Known SLE genes are in bold 
gene not identified in the original studies, also replicated and crossed the false discovery rate (FDR). Two novel genes identified using OASIS were nominally significant, RBFOX1 and MON2/PPM1H.

\section{Gene-based replication}

Both datasets (Harley et al. 2008; Hom et al. 2008) were independently subjected to gene-based association using GATES. Of the 80 candidate genes OASIS identified, 24 nominally replicated using GATES at the gene-based level in at least one dataset (Harley et al. 2008; Hom et al. 2008). IRF5 was the only gene that crossed the Benjamini and Hochberg correction in both datasets (Table 2). Given that known SLE genes from the original studies (Harley et al. 2008; Hom et al. 2008) could not be identified after correction, nominal $P$ values were considered evidence of replication. GATES identified known SLE genes IFIH1 and TNIP1 in dataset phs000122 (Hom et al. 2008) confirming OASIS findings. However,
CD44 did not replicate using GATES in either dataset in spite of being an established SLE gene. Similarly, RBFOX1 also did not replicate using GATES. GRIN2B and $S N X 6$ were the two novel OASIS candidate genes that replicated nominally in both datasets (Harley et al. 2008; Hom et al. 2008). TNFAIP6, DNAJB3, TTF1, MON2, LATS2, NCOA3, and CHAF $1 B$ were verified using GATES in at least one of the datasets (Table 2) (Harley et al. 2008; Hom et al. 2008). Therefore, composite analyses with single-variant replication, gene-based (GATES) and LD clustering (OASIS)-based approaches, have immense potential to mine complex disease genes of low to moderate effect sizes.

\section{Discussion}

In this study, meta-analysis of two dbGAP GWAS datasets (Harley et al. 2008; Hom et al. 2008) using OASIS identified three known SLE genes viz. IFIH1, TNIP1, and CD44 that
Table 2 Gene-based association (GATES) of OASIS candidate genes

\begin{tabular}{|c|c|c|c|c|c|c|}
\hline Chromosome & Position & Gene & $\mathrm{P}^{7}$ & $\mathrm{BH}^{7}$ & $\mathrm{P}^{8}$ & $\mathrm{BH}^{8}$ \\
\hline 2 & $152,214,105$ & TNFAIP6 & & & $3.59 \mathrm{E}-02$ & $8.33 \mathrm{E}-01$ \\
\hline 2 & $163,123,588$ & IFIH1 & & & $3.40 \mathrm{E}-04$ & $3.37 \mathrm{E}-01$ \\
\hline 2 & $191,840,262$ & STAT1 & & & $2.93 \mathrm{E}-02$ & $8.10 \mathrm{E}-01$ \\
\hline 2 & $191,894,301$ & STAT4 & $1.21 \mathrm{E}-03$ & $4.94 \mathrm{E}-01$ & $2.42 \mathrm{E}-12$ & $5.72 E-08$ \\
\hline 2 & $234,651,395$ & DNAJB3 & $7.38 \mathrm{E}-03$ & $6.74 \mathrm{E}-01$ & & \\
\hline 3 & $58,178,352$ & DNASE1L3 & & & $4.25 \mathrm{E}-02$ & $8.51 \mathrm{E}-01$ \\
\hline 3 & $58,318,616$ & PXK & $1.32 \mathrm{E}-02$ & $7.52 \mathrm{E}-01$ & & \\
\hline 5 & $150,409,503$ & TNIP1 & & & $7.31 \mathrm{E}-03$ & $6.45 \mathrm{E}-01$ \\
\hline 7 & $31,823,125$ & PDE1C & & & $1.49 \mathrm{E}-03$ & $4.77 \mathrm{E}-01$ \\
\hline 7 & $128,580,723$ & IRF5 & $2.92 \mathrm{E}-06$ & $1.81 \mathrm{E}-02$ & $8.25 \mathrm{E}-11$ & $9.75 E-07$ \\
\hline 8 & $11,351,520$ & $B L K$ & $9.99 \mathrm{E}-02$ & $9.04 \mathrm{E}-01$ & $2.57 \mathrm{E}-06$ & $1.22 \mathrm{E}-02$ \\
\hline 9 & $135,250,936$ & TTF1 & $1.10 \mathrm{E}-04$ & $1.70 \mathrm{E}-01$ & & \\
\hline 12 & $12,268,960$ & LRP6 & & & $9.80 \mathrm{E}-04$ & $4.51 \mathrm{E}-01$ \\
\hline 12 & $13,714,409$ & GRIN2B & $4.48 \mathrm{E}-02$ & $8.47 \mathrm{E}-01$ & $4.65 \mathrm{E}-02$ & $8.59 \mathrm{E}-01$ \\
\hline 12 & $31,079,837$ & TSPAN11 & $4.80 \mathrm{E}-04$ & $4.71 \mathrm{E}-01$ & & \\
\hline 12 & $62,860,596$ & MON2 & $1.33 \mathrm{E}-02$ & $7.52 \mathrm{E}-01$ & & \\
\hline 13 & $21,547,175$ & LATS2 & & & $1.93 \mathrm{E}-03$ & $5.36 \mathrm{E}-01$ \\
\hline 14 & $35,030,617$ & SNX6 & $4.43 \mathrm{E}-02$ & $8.47 \mathrm{E}-01$ & $4.93 \mathrm{E}-02$ & $8.68 \mathrm{E}-01$ \\
\hline 14 & $73,436,152$ & ZFYVE1 & $1.39 \mathrm{E}-02$ & $7.59 \mathrm{E}-01$ & & \\
\hline 16 & $31,271,287$ & ITGAM & $7.00 \mathrm{E}-04$ & $4.74 \mathrm{E}-01$ & $5.98 \mathrm{E}-06$ & $2.02 E-02$ \\
\hline 16 & $31,366,454$ & ITGAX & $3.35 \mathrm{E}-02$ & $8.29 \mathrm{E}-01$ & $4.81 \mathrm{E}-06$ & $1.90 \mathrm{E}-02$ \\
\hline 20 & $46,130,600$ & NCOA3 & $1.80 \mathrm{E}-03$ & $5.64 \mathrm{E}-01$ & & \\
\hline 20 & $46,286,149$ & $S U L F 2$ & $6.55 \mathrm{E}-03$ & $6.73 \mathrm{E}-01$ & & \\
\hline 21 & $37,757,688$ & $C H A F 1 B$ & & & $7.02 \mathrm{E}-03$ & $6.42 \mathrm{E}-01$ \\
\hline
\end{tabular}

GATES was used to carry out gene-based association of the 80 OASIS candidate genes identified in 30 loci. Known SLE genes and the $p$ values that crossed the Benjamini and Hochberg (BH) correction are highlighted in bold. Nominally significant $p$ values for the genes in the screening (Harley et al. 2008) and replication (Hom et al. 2008) SLE GWAS datasets are shown 
could not be discovered previously using these datasets. The algorithm verified the five genes either of the two SLE studies identified, in both datasets viz. STAT1/STAT4, DNASE1L3/ $P X K, I R F 5, B L K$, and ITGAMIITGAX. Furthermore, 10 new SLE genes were identified and validated using single-variant and gene-based analyses. Hence, OASIS is a unique method of GWAS meta-analysis that can be employed to identify new genes and loci.

Complex disorders such as the SLE are diverse, manifesting more like syndromes than singular diseases (Saeed 2017). Therefore, GWAS cohorts in effect, pool multiple mutations in separate genes of a mixture of resembling phenotypes. For instance, DNAse1L3 mutations code for both SLE and hypocomplementemic urticarial vasculitis (HUVS), phenotypes that are clinically classified separately but nonetheless substantially overlap (Al-Mayouf et al. 2011; Ozçakar et al. 2013). When assumed this way, genome-wide corrections such as Bonferroni lose power to identify the myriad of variants responsible for the phenotypic heterogeneity of a complex disorder. Similarly, more than one gene may exist at a locus for a complex disorder as exemplified by the identification of ANXA6 as a SLE gene located immediately downstream of TNIP1 (Zhang et al. 2015). Hence, LD-based clustering algorithms such as OASIS that focus association signals to loci are of critical importance and can be followed up by biological studies for verification of particular genes.

Despite the identification of large number of genes in multiple comprehensive SLE GWAS and candidate gene studies, they together explain only $15 \%$ of SLE heritability (Bentham et al. 2015). One possible reason for this discrepancy could be increased numbers of false negatives in GWAS due to stringent corrections such as Bonferroni. The $3 \sigma$ rule is a time-tested statistic that, as shown here, can potentially overcome the problem of false negatives in GWAS. OASIS identified BLK and DNAse1L3/PXK loci using the $3 \sigma$ statistic in both SLE datasets, though these had been missed previously in one of the datasets (Harley et al. 2008; Hom et al. 2008). Thus, the $3 \sigma$ statistic applied to GWAS datasets without any correction provides greater opportunity to identify novel genes. Hence, no corrections were applied to the results even though OASIS incorporates two different mechanisms to observe the same underlying phenomenon, i.e., LD (Streiner and Norman 2011). Possibly as this new method of gene discovery evolves, it will become clearer as to what types of correction methods may be applied. However, it has been previously argued that corrections should not be made for multiple comparisons in order to reduce the type II error (Rothman 1990). This was aptly demonstrated in the gene-based replication of OASIS loci using GATES. The only gene that survived the Benjamini and Hochberg correction in both datasets was $I R F 5$, whereas several genes were significant at the uncorrected nominal level (Table 2).
Standard association analysis is based on the $\chi^{2}$ statistic, which is skewed by low-frequency alleles and can result in highly significant $P$ values (type I error). Clustering of significant SNPs, as in OASIS, reduces the possibility of false positive associations (type I error). OASIS is an algorithm that functions in a manner akin to global tests of association such as gene- or pathway-based tests (Christoforou et al. 2012; Neale 2004). The identification in quadrant C, of SLE genes TNIP1 and CD44, validates the novel strategy of LD clustering in OASIS.

Replication has been the classic mechanism of verification to avoid type I errors. Here, the OASIS findings were replicated using standard single-variant and gene-based analyses. The verification of known SLE genes (IFIH1, CD44, and TNIP1/ANXAO) in datasets that did not previously identify them is categorical evidence of the validity of OASIS as a novel gene-hunting tool. About $20 \%$ of variants in OASISidentified loci replicated in single-variant analysis, whereas $30 \%$ of OASIS candidate genes ( 24 of 80 ) replicated using GATES. This together led to the validation of $60 \%$ (18 of 30 ) of the OASIS-identified loci.

These validated SLE genes are biologically relevant. TNFAIP6 codes for the protein TSG-6 which inhibits presentation of chemokines on endothelial surfaces leading to decreased infiltration of T cells and dendritic cells during inflammation (Dyer et al. 2016). Loss-of-function variants in TNFAIP6 may predispose to SLE. TTF1 is a nucleolar factor that controls transcription of the ribosomal RNA genes. This process determines the cell-cycle state from proliferation to apoptosis (Lessard et al. 2012). TTF1 levels are regulated by MDM2-mediated ubiquitinylation (Lessard et al. 2012). MDM2 is known to promote SLE in a murine model (Allam et al. 2011). Possibly, TTFl variants that decrease ubiquitinylation by MDM2 may lead to lymphoproliferation in a manner similar to MDM2 overexpression, leading to SLE (Allam et al. 2011). LATS2 promotes apoptosis by shunting p53 to pro-apoptotic promoters (Aylon et al. 2010). LATS2 is also known to function as a negative regulator of TNF- $\alpha$ induced NF-KB activation (Yao et al. 2015). CHAF1B is involved in epigenetic control of chromatin dynamics during cell cycling, and its inhibition leads to apoptosis and accumulation of double-strand breaks (Nabatiyan and Krude 2004). MON2 and SNX6 are involved in endosome-to-Golgi trafficking (Mahajan et al. 2013). SNX6 traffics members of the TGF- $\beta$ family of receptor serine-threonine kinases (Parks et al. 2001). NCOA3 codes for the SRC-3 protein that plays an important role in maintenance of $\mathrm{T}$ cell function ( $\mathrm{Li}$ et al. 2012).

The OASIS algorithm provides an alternative to increasing sample sizes for GWAS to ascertain variants with low to moderate effect sizes. This is made possible by composite analysis based on two axes (-log $[\mathrm{P}]$ and OASIS blocks) divided into association quadrants. This unifies two aspects of the LD 
phenomenon - strength of association of a single-variant and the number of significant genetic markers (Fig. S1). Caveats with the algorithm are that the results may be affected by population-based LD, leading to high OASIS scores in regions such as the HLA genomic segments (Fig. 1). Moreover, the number of SNPs genotyped at a locus may skew the scores as well. Population stratification in case control studies will likely affect OASIS results as it does standard association analysis.

In summary, OASIS is a novel LD clustering algorithm described here that can be widely applied for mining existing GWAS datasets to identify candidate genes in an efficient, low-cost way. These candidates can be subsequently replicated in other studies such as single-variant, gene-based analyses and biological studies. Here, OASIS was applied to two dbGAP SLE GWAS datasets and identified 8 known and 10 novel SLE genes. OASIS was verified using two sets of analysis viz. single-variant replication and gene-based analyses using GATES. This study also underscores the need to make more GWAS datasets publically available for further development of novel analytic tools. Taken together, these findings highlight the novelty and efficiency of LD-based clustering approaches, such as the OASIS algorithm, for GWAS metaanalysis of complex disorders.

\section{Compliance with ethical standards}

Conflict of interest The author declares that he has no conflict of interest.

\section{Funding None}

Open Access This article is distributed under the terms of the Creative Commons Attribution 4.0 International License (http:// creativecommons.org/licenses/by/4.0/), which permits unrestricted use, distribution, and reproduction in any medium, provided you give appropriate credit to the original author(s) and the source, provide a link to the Creative Commons license, and indicate if changes were made.

\section{References}

Allam R, Sayyed SG, Kulkarni OP, Lichtnekert J, Anders HJ (2011) $\mathrm{Mdm} 2$ promotes systemic lupus erythematosus and lupus nephritis. J Am Soc Nephrol 22(11):2016-2027

Al-Mayouf SM, Sunker A, Abdwani R, Abrawi SA, Almurshedi F, Alhashmi N, Al Sonbul A et al (2011) Loss-of-function variant in DNASE1L3 causes a familial form of systemic lupus erythematosus. Nat Genet 43(12):1186-1188

Aylon Y, Ofir-Rosenfeld Y, Yabuta N, Lapi E, Nojima H, Lu X, Oren M (2010) The Lats2 tumor suppressor augments p53-mediated apoptosis by promoting the nuclear proapoptotic function of ASPP1. Genes Dev 24(21):2420-2429

Bentham J, Morris DL, Cunninghame Graham DS, Pinder CL, Tombleson P, Behrens TW, Martín J et al (2015) Genetic association analyses implicate aberrant regulation of innate and adaptive immunity genes in the pathogenesis of systemic lupus erythematosus. Nat Genet 47(12):1457-1464
Christoforou A, Dondrup M, Mattingsdal M, Mattheisen M, Giddaluru S, Nöthen MM, Rietschel M et al (2012) Linkage-disequilibriumbased binning affects the interpretation of GWASs. Am J Hum Genet 90(4):727-733

Duerr RH, Taylor KD, Brant SR, Rioux JD, Silverberg MS, Daly MJ, Steinhart AH et al (2006) A genome-wide association study identifies IL23R as an inflammatory bowel disease gene. Science 314(5804):1461-1463 PMID: 17068223

Dyer DP, Salanga CL, Johns SC, Valdambrini E, Fuster MM, Milner CM, Day AJ et al (2016) The anti-inflammatory protein TSG-6 regulates chemokine function by inhibiting chemokine/glycosaminoglycan interactions. J Biol Chem 291(24):12627-12640

Edwards AO, Ritter R 3rd, Abel KJ, Manning A, Panhuysen C, Farrer LA (2005) Complement factor $\mathrm{H}$ polymorphism and age-related macular degeneration. Science 308(5720):421-424

Gateva V, Sandling JK, Hom G, Taylor KE, Chung SA, Sun X, Ortmann $\mathrm{W}$ et al (2009) A large-scale replication study identifies TNIP1, PRDM1, JAZF1, UHRF1BP1 and IL10 as risk loci for systemic lupus erythematosus. Nat Genet 41(11):1228-1233

Harley JB, Alarcón-Riquelme ME, Criswell LA, Jacob CO, Kimberly RP, Moser KL, Tsao BP, International Consortium for Systemic Lupus Erythematosus Genetics (SLEGEN) et al (2008) Genome-wide association scan in women with systemic lupus erythematosus identifies susceptibility variants in ITGAM, PXK, KIAA1542 and other loci. Nat Genet 40(2):204-210

Hom G, Graham RR, Modrek B, Taylor KE, Ortmann W, Garnier S, Lee AT et al (2008) Association of systemic lupus erythematosus with C8orf13-BLK and ITGAM-ITGAX. N Engl J Med 358(9):900-909

Lessard CJ, Adrianto I, Kelly JA, Kaufman KM, Grundahl KM, Adler A, Williams AH et al (2011) Identification of a systemic lupus erythematosus susceptibility locus at 11p13 between PDHX and CD44 in a multiethnic study. Am J Hum Genet 88(1):83-91

Lessard F, Stefanovsky V, Tremblay MG, Moss T (2012) The cellular abundance of the essential transcription termination factor TTF-I regulates ribosome biogenesis and is determined by MDM2 ubiquitinylation. Nucleic Acids Res 40(12):5357-5367

Li J, Niu J, Ou S, Ye ZY, Liu DQ, Wang FC, Su YP, Wang JP (2012) Effects of SCR-3 on the immunosuppression accompanied with the systemic inflammatory response syndrome. Mol Cell Biochem 364(1-2):29-37

Li MX, Gui HS, Kwan JS, Sham PC (2011) GATES: a rapid and powerful gene-based association test using extended Simes procedure. Am J Hum Genet 88(3):283-293

Li MX, Sham PC, Cherny SS, Song YQ (2010) A knowledge-based weighting framework to boost the power of genome-wide association studies. PLoS One 5(12):e14480

Mahajan D, Boh BK, Zhou Y, Chen L, Cornvik TC, Hong W, Lu L (2013) Mammalian Mon2/Ysl2 regulates endosome-to-Golgi trafficking but possesses no guanine nucleotide exchange activity toward Arl1 GTPase. Sci Rep 3:3362

Martin ER, Lai EH, Gilbert JR, Rogala AR, Afshari AJ, Riley J, Finch KL et al (2000) SNPing away at complex diseases: analysis of singlenucleotide polymorphisms around APOE in Alzheimer disease. Am J Hum Genet 67(2):383-394 PMID: 10869235

Nabatiyan A, Krude T (2004) Silencing of chromatin assembly factor 1 in human cells leads to cell death and loss of chromatin assembly during DNA synthesis. Mol Cell Biol 24(7):2853-2862

Neale BM (2004) Sham PC. The future of association studies: gene-based analysis and replication. Am J Hum Genet 75(3):353-362

Ozçakar ZB, Foster J 2nd, Diaz-Horta O, Kasapcopur O, Fan YS, Yalçınkaya F, Tekin M (2013) DNASE1L3 mutations in hypocomplementemic urticarial vasculitis syndrome. Arthritis Rheum 65(8):2183-2189

Parks WT, Frank DB, Huff C, Renfrew Haft C, Martin J, Meng X, de Caestecker MP et al (2001) Sorting nexin 6, a novel SNX, interacts 
with the transforming growth factor-beta family of receptor serinethreonine kinases. J Biol Chem 276(22):19332-19339

Ramos PS, Williams AH, Ziegler JT, Comeau ME, Guy RT, Lessard CJ, Li H et al (2011) Genetic analyses of interferon pathway-related genes reveal multiple new loci associated with systemic lupus erythematosus. Arthritis Rheum 63(7):2049-2057

Rioux JD, Xavier RJ, Taylor KD, Silverberg MS, Goyette P, Huett A, Green $T$ et al (2007) Genome-wide association study identifies new susceptibility loci for Crohn disease and implicates autophagy in disease pathogenesis. Nat Genet 39(5):596-604

Robinson T, Kariuki SN, Franek BS, Kumabe M, Kumar AA, Badaracco M, Mikolaitis RA et al (2011) Autoimmune disease risk variant of IFIH1 is associated with increased sensitivity to IFN- $\alpha$ and serologic autoimmunity in lupus patients. J Immunol 187(3):1298-1303

Rothman KJ (1990) No adjustments are needed for multiple comparisons. Epidemiology 1(1):43-46

Saeed M, Yang Y, Deng HX, Hung WY, Siddique N, Dellefave L, Gellera C, Andersen PM, Siddique T (2009) Age and founder effect of SOD1 A4V mutation causing ALS. Neurology 72(19):1634-1639

Saeed M (2017) Lupus pathobiology based on genomics. Immunogenetics 69(1):1-12
Sheng YJ, Xu JH, Wu YG, Zuo XB, Gao JP, Lin Y, Zhu ZW et al (2015) Association analyses confirm five susceptibility loci for systemic lupus erythematosus in the Han Chinese population. Arthritis Res Ther 17:85

Streiner DL, Norman GR (2011) Correction for multiple testing: is there a resolution? Chest 140(1):16-18

Wang C, Ahlford A, Laxman N, Nordmark G, Eloranta ML, Gunnarsson I, Svenungsson E et al (2013) Contribution of IKBKE and IFIH1 gene variants to SLE susceptibility. Genes Immun 14(4):217-222

Yao F, Zhou W, Zhong C, Fang W (2015) LATS2 inhibits the activity of NF-K B signaling by disrupting the interaction between TAK1 and IKK $\beta$. Tumour Biol 36(10):7873-7879

Yung S, Chan TM (2012) The role of hyaluronan and CD44 in the pathogenesis of lupus nephritis. Autoimmune Dis 2012:207190

Zhang J, Zhang L, Zhang Y, Yang J, Guo M, Sun L, Pan HF et al (2015) Gene-based meta-analysis of genome-wide association study data identifies independent single-nucleotide polymorphisms in ANXA6 as being associated with systemic lupus erythematosus in Asian populations. Arthritis Rheumatol 67(11):2966-2977 